\title{
Photonic generation of broadband RF phase shift with unbounded phase trajectory
}

This paper was downloaded from TechRxiv (https://www.techrxiv.org).

\section{LICENSE}

CC BY 4.0

SUBMISSION DATE / POSTED DATE

$26-01-2021 / 02-02-2021$

CITATION

Hasan, Mehedi; hall, trevor (2021): Photonic generation of broadband RF phase shift with unbounded phase trajectory. TechRxiv. Preprint. https://doi.org/10.36227/techrxiv.13641533.v1

DOI

10.36227/techrxiv.13641533.v1 


\title{
Photonic generation of broadband RF phase shift with unbounded phase trajectory
}

\author{
MEHEDI HASAN, ${ }^{1, *}$ AND TREVOR HALL ${ }^{1}$ \\ ${ }^{1}$ Photonic Technology Laboratory, Centre for Research in Photonics, Advanced Research Complex, University of \\ Ottawa, 25 Templeton Street, Ottawa, K1N 6N5, ON, Canada \\ *mhasa067@uottawa.ca
}

\begin{abstract}
A means of applying an adjustable RF phase shift over a broad band of frequencies is a requirement of diverse application. Photonic solutions to the generation of RF phase shifts have receive significant attention for reasons of reduced cost, compactness and simplicity, yet the achievement of a phase shift extending beyond $360^{\circ}$ range remains a challenge. The circuit architecture of a compact and broadband RF phase shifter with unbounded range based on two parallel DP-MZM architecture is presented and verified by simulation verification and emulated using off the shelf low frequency electronic components. Results demonstrate that the complex transmission of the phase shifter follows a trajectory that may encircle the origin an arbitrary number of times in either direction. The proposed architecture can be implemented using commercially available DP-QPSK modulator or can be integrated in any material platform that offers linear electro-optic phase modulators.
\end{abstract}

\section{Introduction}

A precise and broadband radio frequency (RF) phase shifter is needed in a variety of applications including phased array antenna systems to support high speed wireless communication and radar; and self interference cancellation (SIC) systems in a full duplex technology to improve the attainable spectral efficiency of a 5G network. The photonic generation of microwave phase shifts is receiving significant attention due to its simplicity, light weight, low cost, small size and broadband operation with minimal phase errors. The underlying principle is based on frequency shifting an optical carrier via single side band suppressed carrier (SSB-SC) modulation using RF carrier of interest followed by the re-insertion of a copy of the original optical carrier that is shifted in phase by a variety of means such as an optical phase modulator based on p-n junction devices; z-cut lithium niobate (LiNbO3); or a polarization controller within a number of different circuit architectures [1-8].

In reference [1] an optical carrier is divided in to two paths, one used to generate SSB-SC modulation, while the other path is phase shifted using an optical phase modulator. Both the paths are then combined on a photodiode to generate the phase shifted RF signal. A similar strategy to obtain the phase shift is followed in references [2-4] using a dual-polarization dual-parallel Mach Zehnder Modulator (DP-MZM); in which one DP-MZM generates the SSBSC modulation while the other DP-MZM is kept unmodulated and arranged to provide a standard linear phase shift of the unmodulated carrier with limited range. The outputs of the both DP-MZM are then combined using a polarization beam combiner (PBC). The relative phase of the orthogonally polarised optical carrier and sideband is then adjusted by a polarization dependent optical phase shifter (OPS) [2] or a combination of polarization controller and polarizer [3-4]. A DP-MZM based scheme is presented in [5-8]. In [5], one child MZM is biased at the minimum transmission point (MITP) and driven by an RF signal, thereby generating double sideband suppress carrier (DSB-SC) modulation; while the other child MZM is biased at the MATP to pass the carrier. The two outputs are combined by using an optical coupler [6]. The optical phase shift is applied by tuning the phase adjust voltage of the parent MZM. Finally, an optical bandpass filter (OBF) is used to suppress one of the sidebands. A similar method is described in [6] except the combining is done through a PBC and the optical phase shift is implemented using polarization dependent OPS. Reference [7] describes a DP-MZM based scheme capable of a $360^{\circ}$-degree phase shift founded on the precise settings of the phase and amplitude of the optical carrier and two sidebands using predetermined modulator bias voltages. An architecture consisting of, two parallel MZM driven by in-phase and quadrature phase RF signal modulate orthogonally polarised optical carriers combined on a photodiode is demonstrated in [8]. The slope about the operating point of the two MZM is varied by adjustment of their respective DC bias voltage, thereby the sign and magnitude of the in-phase and quadrature phase modulation components is adjusted resulting in the phase shift of the superimposed components. For proper operation of the scheme, the modulation depth should be small which leads to high insertion loss and careful adjustment of the two bias voltages is required as, for a constant magnitude RF output, both bias voltages depend on the desired phase shift. A photonic integrated phase shifter based on silicon-on-insulator technology is presented in [9]. A single side band (SSB) full carrier modulation is generated using a DP-MZM. An optical deinterleaver filter is used to separate the optical carrier and the sideband. The phase of the isolated carrier is then shifted by using a p-n junction based optical phase shifter and combined with the sideband on a photodiode. All 
these methods require either an optical deinterleaver filter or optical bandpass filter, precise control of the polarization and/or polarizer, bias voltages or suffers from lack of phase shift linearity and range. Moreover, the need for a continuous phase shift range extending beyond $360^{\circ}$ degree is gaining appreciation. For an example, improved robustness to loss of lock of an optoelectronic oscillator has been demonstrated by enlarging the RF tuning phase shift ranges from $350^{\circ}$ degrees to $1160^{\circ}$ degree using a frequency conversion pair [10].

In this paper, a circuit architecture is proposed based on a parallel pair of dual-parallel Mach-Zehnder modulators (DP-MZM) that requires no additional control or filter for proper operation. Furthermore, the linearity of the phase shifter remains valid for a wide range of frequencies. The proposed phase shifter can generate any phase without bound. The complex transmission of the phase shifter follows a trajectory in the complex plane that may encircle the origin an arbitrary number of times in either direction. The practical implementation of the proposed design can be demonstrated using a commercially available dual polarization quadrature phase shift keying (DP-QPSK) modulator. Due to unavailability of a-QPSK modulator, experimental verification by an electronic emulation of the proposed concept is presented using off the shelf components.

\section{Principal of operation}

The circuit diagram of the proposed braodband phase shifter is given in fig. 1. The circuit consists of two DP-MZM. The upper DP-MZM generates the suppressed carrier-single side band modulation of the applied RF drive signal. To obtain this function, the bias of the two parallel child MZM is set to their minmum tranmission points (MITP), while the mother MZM is set to its quadrature transmission point (QTP). Then the in-phase (I) and quadrature-phase (Q) of the RF drive signal is applied to the upper and lower child MZM respectively [11]. SSB-SC modulation can also be regarded as the shifting of the optical carrier frequency by an amount equal to the RF frequency. The bias of the lower DP-MZM is set similarly to the upper DP-MZM, i.e the two parallel MZM is set to MITP or null transmission point and the mother MZM is set to QTP. A static or low frequency in-phase and quadrature-phase signal is applied to the two child MZM to shift the phase of the optical carrier. When the output of the two DP-MZM imping on a photodiode, a phase shifted version of the RF drive signal is recovered.
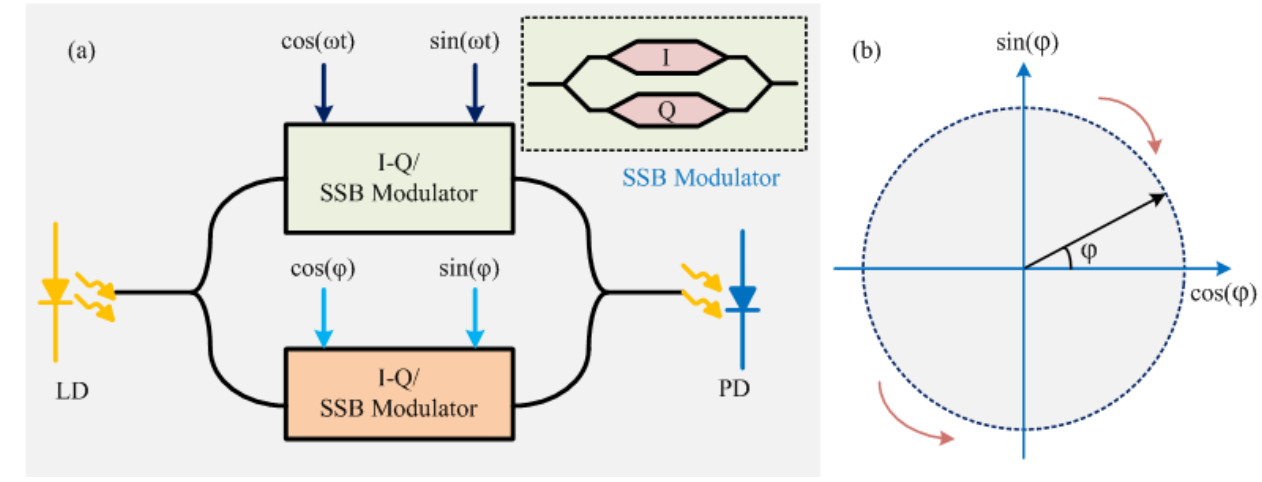

Fig. 1. (a) Schematic diagram of the proposed broadband phase shifter; (b) phase shift trajectory (either clockwise or anticlockwise); LD, Laser diode; SSB, Single side band; PD, Photodiode.

In the small modulation index $\left(m_{1}\right)$ approximation, the transmission [11] of the upper DP-MZM is

$$
T_{\text {upper } D P-M Z M}=(1 / 4) m_{1} \exp \left(i\left(\omega_{c} \pm \omega_{r f}\right) t\right)
$$

where, $\omega_{c}$ and $\omega_{r f}$ is the optical and RF carrier frequency. Depending on the phase lead-lag between upper MZM and lower MZM, either an upper SSB-SC or lower SSB-SC modulation is obtained. Alternatively, one output port generates either upper/lower SSB-SC modulation while the other output port generates the complementary SSB-SC modulation for a two-output port DP-MZM [12]. The modulation index $m_{1}$ is defined by:

$$
m_{1}=\pi v_{R F} / v_{\pi}
$$

where, $v_{R F}$ is the amplitude of the applied RF signal and $v_{\pi}$ is the half-wave voltage of the phase modulator used to form the MZM. Similar way, the output transmission of the lower DP-MZM can be written: 


$$
T_{\text {Lower } D P-M Z M}=(1 / 4) m_{2} \exp \left(i\left(\omega_{c} t \pm \varphi\right)\right)
$$

where $\varphi$ is the applied phase shift to the optical carrier through I-Q modulation at the lower DP-MZM. The modulation index $m_{2}$ can be written as:

$$
m_{2}=\pi v_{p} / v_{\pi} \quad \text { where } \quad v_{p}=\sqrt{v_{I}^{2}+v_{Q}^{2}}
$$

The $v_{\pi}$ here may be different than for $m_{1}$ due to the difference in the frequency of the RF modulation and the IQ controls. The output of the upper DP-MZM and lower DP-MZM is combined and impinged to a photodiode. Finally, the beat signal of interest at the output of the photodiode is,

$$
\propto \cos \left(\omega_{r f} \tau \mp\right)
$$

The phase shift $\varphi$ of the RF signal given by Eq. (4) may be continuously varied by a continuous variation of the bounded I and Q bias at the lower DP-MZM. The small modulation index $m_{1}$ is a non-necessary condition. Jacobi Anger expansion leads to same solution while providing details on the amplitude of the Bessel sidebands [13].

Rather than applying the IQ RF and IQ bias separately to a parallel pair of IQ modulators, the vector sum of the IQ RF drive and IQ bias may be applied to a single IQ modulator to obtain the same result to the extent that an IQ modulator has a transmission is linear with respect to the IQ vector. Consequently, a single DP-MZM can be used to perform a similar operation [14]. However, without special linearization [15] measures the single DP-MZM arrangement is limited to small modulation indices $m_{1}, m_{2}$ resulting in high insertion loss compared to the parallel DP-MZM solution.

\section{Simulation verification $\&$ experimental result}

Circuit simulation using VPIphotonics is performed to verify the theoretical prediction. A Distributed Feedback (DFB) laser having wavelength of $1550 \mathrm{~nm}$ and an output power of $10 \mathrm{dBm}$ is used as the optical source. The insertion loss and extinction ratio of each MZM is set to be $4 \mathrm{~dB}$ and $30 \mathrm{~dB}$ respectively. The half-wave voltage of each MZM is set to $5 \mathrm{~V}$. The in-phase and quadrature phase of the RF drive signal with peak amplitude of $1.2 \mathrm{~V}$ and a frequency of 10 $\mathrm{GHz}$ is applied to the upper DP-MZM. This results in a frequency shift of the optical carrier by an amount of $10 \mathrm{GHz}$. Although suppressed-carrier modulation is desired, the suppression is not complete due to the finite extinction of the MZM. For the purpose of demonstrating the operating principle by simulation, a very low frequency cosine- and sinewaveform are applied to the in-phase and quadrature-phase inputs to the lower DP-MZM to generate a slow linear ramp of the optical carrier phase. Once both the output of the upper and lower DP-MZM are combined at the photodiode, the desired phase shifted RF carrier can be observed.

Figure 2 shows the phase shift at different static phase $(\varphi)$ of the low frequency drive signal predicted by the simulation. The output phase shift exactly matches the applied phase shift and without bound. The corresponding phasor can rotate about the origin of the complex plane in either direction (clockwise or anti-clockwise) an arbitrary number of times. The power variation of the recovered signal at different phase shifts is found to be $\sim 1.8 \mathrm{~dB}$ maximum. This variation is the result of the nonlinear transmission function of the DP-MZM and may be corrected by predistortion of the in-phase and quadrature phase controls of the lower DP-MZM or by application of alternative methods [15] of the lower DP-MZM. The proposed circuit can be used up to the bandwidth of the phase modulators used to

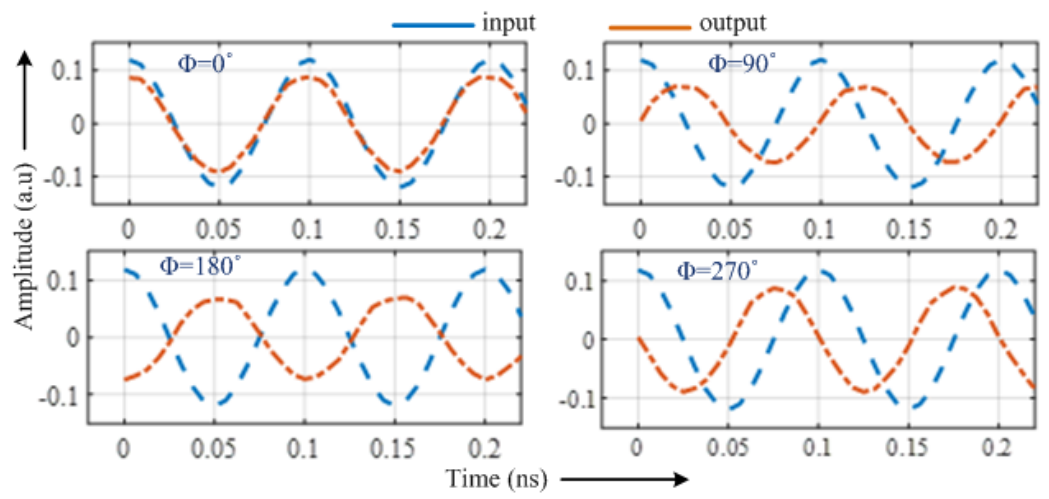

Fig. 2. Simulated phase relationship between the input and output RF signal. 
form the DP-MZM. The practical implementation of the proposed circuit can be realized by commercially available dual polarization quadrature phase shift keying (DP-QPSK) modulator. Hybrid integration of silica and modulator based on $\mathrm{LiNbO} 3$ can be used to verify the concept [16].

Due to unavailability of a DP-QPSK-modulator and vector network analyzer, the concept is verified experimentally using off the shelf lower frequency electronic components. Figure 3 shows the schematic diagram of the experimental setup. An Analog Devices HMC630 used as vector modulator to emulate the function of a DP-MZM. The vector modulator has one input port, one output port and two other input ports for the application of I and Q controls. The operating frequency range is $700-1000 \mathrm{MHz}$, whereas the $3 \mathrm{~dB}$ bandwidth of the I and Q ports are 180 MHz. A Minicircuits ADP-2-10W (5 to $1000 \mathrm{MHz}$ ) is used as the input power splitter. To emulate the function of a photodiode, a double balanced mixer (ADE-2+) from Minicircuits is used. A RF signal with a frequency of $800 \mathrm{MHz}$ and $10.5 \mathrm{dBm}$ output power is used as the input carrier. A Rohde \& Schwarz SMB 100A signal generator is used as the RF LO. An IF signal having frequency of $115 \mathrm{MHz}$ is applied to a $90^{\circ}$ hybrid (Minicircuits ADQ-22+) to generate the in-phase and quadrature-phase components. The in-phase and quadrature-phase component is then applied to the I and Q ports respectively of the upper vector modulator.

Figure 3(b) shows the measured electrical spectrum of the SSB-SC modulation generated at a frequency $915 \mathrm{MHz}$. The sideband (at $915 \mathrm{MHz}$ ) to carrier (at $800 \mathrm{MHz}$ ) suppression ratio is $15 \mathrm{~dB}$. In an optical implementation, the RF LO is replaced by an optical carrier and the IF signal is by RF signal that is needed to be phase shifted. In optics, a

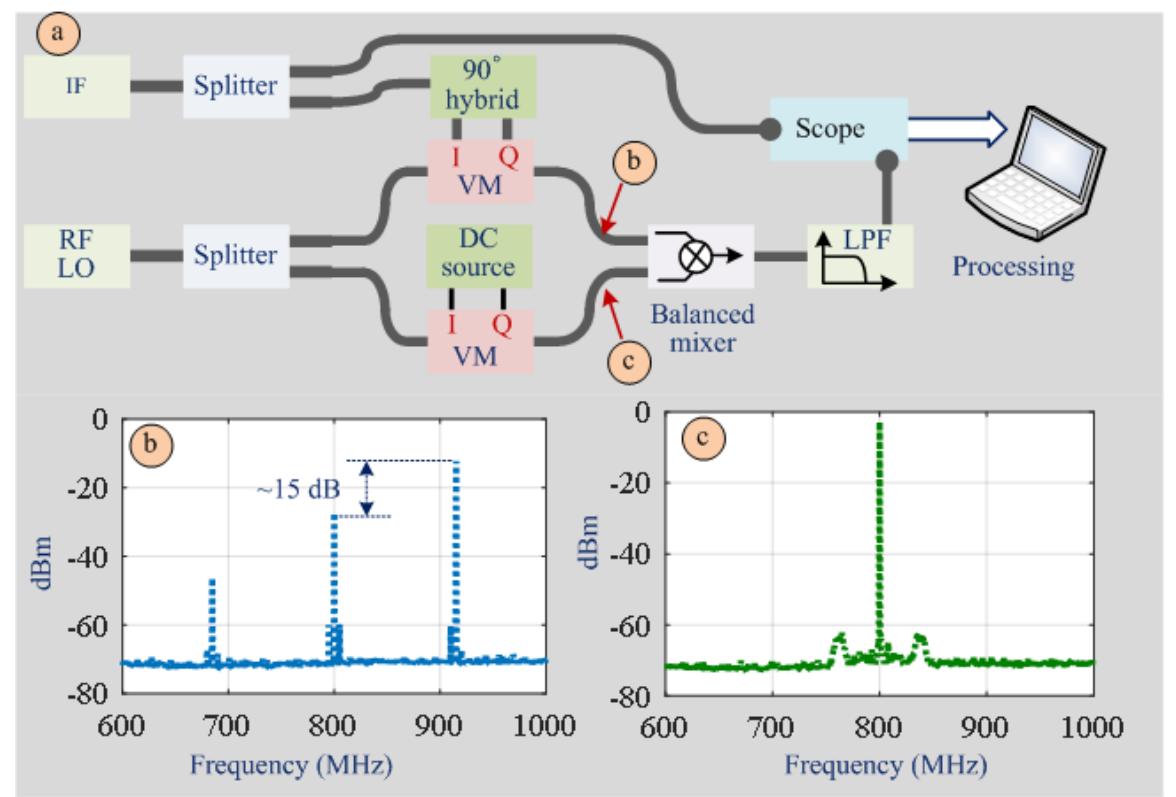

Fig. 3. (a) Schematic of the experimental setup; (b) and (c) are the measured spectra (electrical) of the signal at point (b) and (c) in schematic (a); IF, intermediate frequency; LO, local oscillator; VM, vector modulator; DC, direct current. An electrical resolution bandwidth of $1 \mathrm{kHz}$ is used to capture the data.

sideband to carrier suppression ratio of $\sim 20-22 \mathrm{~dB}$ was reported experimentally using a DP-MZM architecture [1213] a decade ago. A suppression of $\sim 40 \mathrm{~dB}$ is obtained [16] using a phase shifter and/or variable optical attenuator (VOA) as a trimming means. The phase of the carrier that passes through the lower vector modulator is adjusted by applying a DC voltage in the range of $0.5 \mathrm{~V}$ to $2.5 \mathrm{~V}$ to the $\mathrm{I}$ and $\mathrm{Q}$ port of the lower vector modulator using a precision DC source (Agilent B2912A). To adhere the specified ranges, the applied I and Q voltages are defined by:

$$
\begin{aligned}
& I=1.5+\cos (\varphi) \\
& Q=1.5+\sin (\varphi)
\end{aligned}
$$




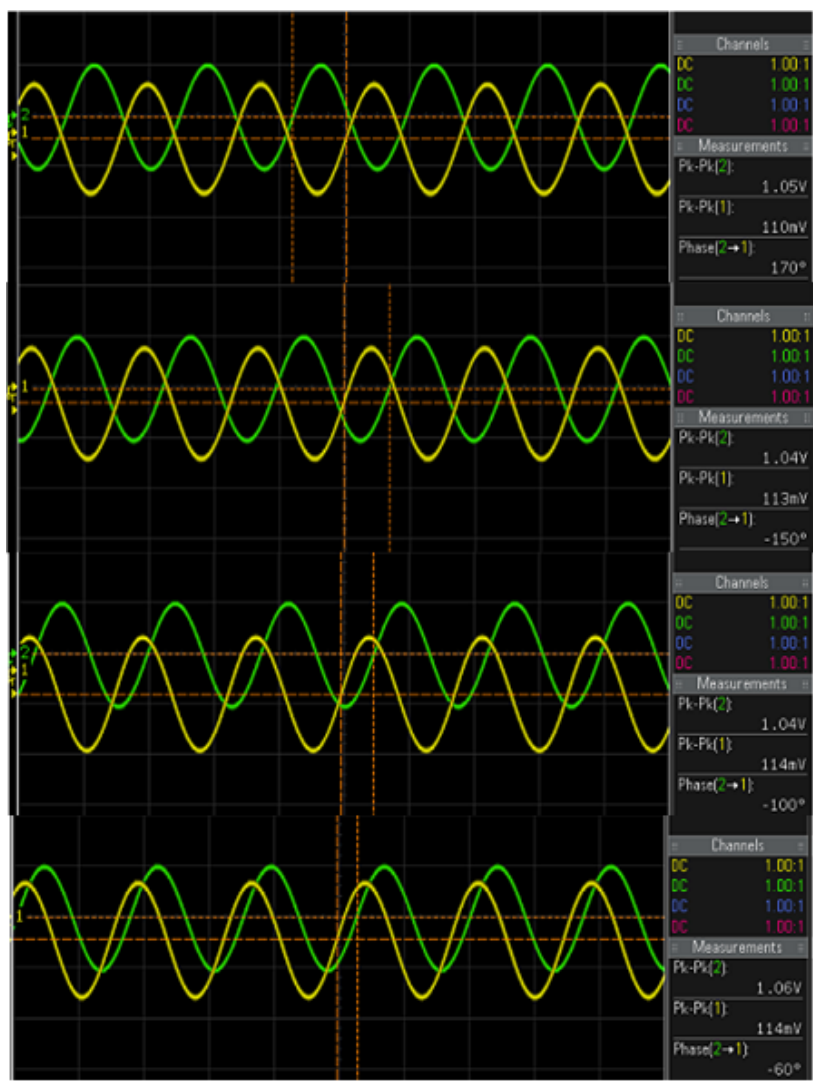

(a). $\quad \mathrm{I}=1.5+\cos \left(0^{\circ}\right)$

$\mathrm{Q}=1.5+\sin \left(0^{\circ}\right)$

Measured phase $=170^{\circ}$

Adjusted phase $=0^{\circ}$

Actual phase shift $=0^{\circ}$

(b). $\quad \mathrm{I}=1.5+\cos \left(45^{\circ}\right)$

$\mathrm{Q}=1.5+\sin \left(45^{\circ}\right)$

Measured phase $=-150^{\circ}$

Adjusted phase $=-320^{\circ}$

Actual phase shift $=40^{\circ}$

(c). $I=1.5+\cos \left(90^{\circ}\right)$

$\mathrm{Q}=1.5+\sin \left(90^{\circ}\right)$

Measured phase $=-100^{\circ}$

Adjusted phase $=-270^{\circ}$

Actual phase shift $=90^{\circ}$

(d). $\quad I=1.5+\cos \left(135^{\circ}\right)$

$\mathrm{Q}=1.5+\sin \left(135^{\circ}\right)$

Measured phase $=-60^{\circ}$

Adjusted phase $=-230^{\circ}$

Actual phase shift $=130^{\circ}$

Fig. 4. Screen image of the oscilloscope showing the phase difference between the generated RF signal and a reference signal for various target phase shift as input; (a) target phase shift $0^{\circ}$; (b) target phase shift $45^{\circ}$; (c) target phase shift $90^{\circ}$ and (d) $135^{\circ}$ respectively.

A phase ramp is applied to the carrier by changing the value of $\varphi$, results in equal amount of phase shift at the recovered IF signal at the output of the mixer. Figure 3(c) shows the electrical spectrum of the measured carrier for a particular combination of I and Q values. A similar length of semi rigid RF cable is used to connect the two vector modulators with the double balanced mixer, making sure that the path lengths remain same. In a photonic integrated circuit, this can easily be achieved. The output of the mixer is then passed through a low pass filter (LPF) to the digital storage oscilloscope. Since, there is no vector network analyzer (VNA), the length of connection between the LPF and oscilloscope is kept to a minimum so that the actual phase at the output of the LPF is measured by the oscilloscope. Nevertheless, an error of $\sim 2-3^{\circ}$ is added due to the different path lengths. Figure 4 and 5 shows the screen image of

Table 1. Experimental details of the applied input voltage (target phase), measured phase, actual phase shift and peak to peak voltage of the recovered IF signal.

\begin{tabular}{|l|l|c|c|c|c|c|}
\hline \multicolumn{2}{|c|}{$\begin{array}{c}\text { Input voltage } \\
\text { (volts) }\end{array}$} & $\begin{array}{c}\text { Target } \\
\text { phase } \\
\text { degree }\end{array}$ & $\begin{array}{c}\text { Phase shift with } \\
\text { respect to reference } \\
\text { signal (degree) }\end{array}$ & $\begin{array}{c}\text { Adjusted phase } \\
\text { (degree) }\end{array}$ & $\begin{array}{c}\text { Actual } \\
\text { phase shift } \\
\text { (degree) }\end{array}$ & $\begin{array}{l}\text { Amplitude } \\
V_{\text {peak-peak }} \\
(\mathrm{mV})\end{array}$ \\
\cline { 1 - 2 } I-port & Q-port & 0 & 170 & $170-170=0$ & 0 & 110 \\
\hline 2.5 & 1.5 & 25 & -170 & $-170-170=-340$ & 20 & 111 \\
\hline 2.406 & 1.922 & 45 & -150 & $-150-170=-320$ & 40 & 113 \\
\hline 2.207 & 2.207 & 90 & -100 & $-100-170=-270$ & 90 & 114 \\
\hline 1.5 & 2.5 & 135 & -60 & $-60-170=-230$ & 130 & 114 \\
\hline 0.793 & 2.207 & 180 & -19 & $-19-170=-189$ & 171 & 112 \\
\hline 0.5 & 1.5 & 210 & 10 & $10-170=-160$ & 200 & 110 \\
\hline 0.633 & 1.0 & 225 & 29 & $29-170=-141$ & 219 & 109 \\
\hline 0.793 & 0.793 & 245 & 50 & $50-170=-120$ & 240 & 110 \\
\hline 1 & 0.633 & 270 & 80 & $80-170=-90$ & 270 & 112 \\
\hline 1.5 & 0.5 & 315 & 120 & $120-170=-50$ & 310 & 110 \\
\hline 2.207 & 0.793 & 335 & 140 & $140-170=-30$ & 330 & 110 \\
\hline 2.406 & 1.077 & & & & \\
\hline
\end{tabular}




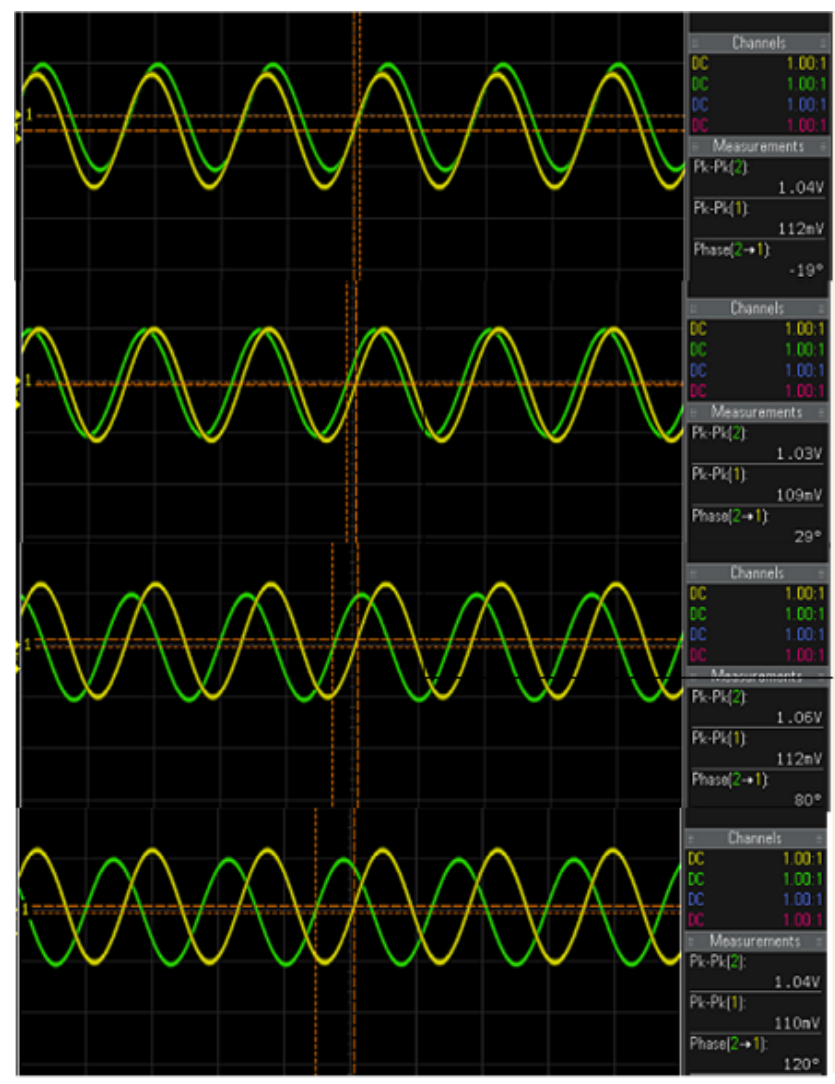

(a). $I=1.5+\cos \left(180^{\circ}\right)$

$\mathrm{Q}=1.5+\sin \left(180^{\circ}\right)$

Measured phase $=-19^{\circ}$

Adjusted phase $=-189^{\circ}$

Actual phase shift $=171^{\circ}$

(b). $\quad \mathrm{I}=1.5+\cos \left(225^{\circ}\right)$

$\mathrm{Q}=1.5+\sin \left(225^{\circ}\right)$

Measured phase $=29^{\circ}$

Adjusted phase $=-141^{\circ}$

Actual phase shift $=219^{\circ}$

(c). I $=1.5+\cos \left(270^{\circ}\right)$

$\mathrm{Q}=1.5+\sin \left(270^{\circ}\right)$

Measured phase $=80^{\circ}$

Adjusted phase $=-90^{\circ}$

Actual phase shift $=270^{\circ}$

(d). $\quad I=1.5+\cos \left(315^{\circ}\right)$

$\mathrm{Q}=1.5+\sin \left(315^{\circ}\right)$

Measured phase $=120^{\circ}$

Adjusted phase $=-50^{\circ}$

Actual phase shift $=310^{\circ}$

Fig. 5. Screen image of the oscilloscope showing the phase difference between the generated RF signal and a reference signal for various target phase shift as input; (a) target phase shift $180^{\circ}$; (b) target phase shift $225^{\circ}$; (c) target phase shift $270^{\circ}$ and (d) $315^{\circ}$ respectively.

the measured phase difference between the recovered IF signal and the reference signal for various input phase values $\varphi$. The measured phase difference is then adjusted by subtracting an equal amount of phase shift from all the measured phase to obtain the actual phase shift. Table 1 shows the details of applied voltage to the I and Q port, measured phase difference and actual phase shift. Figure 6. (a) plots the measured phase shift as a function of applied phase shift (target phase shift). A straight line is obtained using curve fitting which also justify the theoretical prediction. A very small error is obtained between the target phase shift and actual phase shift. In some cases, the measured phase shift is identical to the target shift. Measurement results also verify that the peak to peak amplitude of the recovered IF signal remain almost constant. Figure 6. (b) shows the comparison between expected I-Q values and measured I-Q values. A maximum error of $9-10^{\circ}$ degree is obtained for two cases. However, the phase measurement using a VNA will certainly improve the precision of the measurement. Nevertheless, a $360^{\circ}$-degree rotation is obtained experimentally.
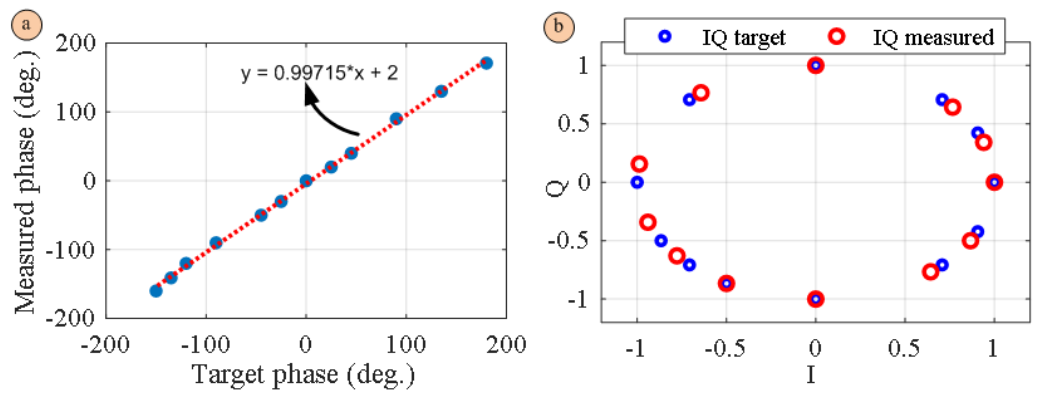

Fig. 6. (a) Relationship between target and measured phase shift; (b) measured I-Q value versus expected I-Q value. 
The phase trajectory can rotate on the unit circle either way (clockwise or anti clockwise) indefinite amount of times.

\section{Conclusion}

In summary, a novel photonic technique for obtaining the phase shift of a RF signal is reported with simulation verification. The proposed circuit can function for a wide range of frequencies and it can generate a phase without bound. The circuit may be implemented in practice using a commercially available DP-QPSK modulator. The bandwidth of the operation depends on the bandwidth of the phase modulators that forms the MZM. An experimental verification of the concept is presented using off the shelf low frequency electronics. Results show that a RF phase shift of $360^{\circ}$ degree or more can be obtained with negligible penalty at the amplitude of the phase shifted signal. Results also show that an SSB-SC modulation having sideband to carrier suppression ratio of only $15 \mathrm{~dB}$ or more is good enough for the proposed architecture to function correctly

\section{Funding}

No funding.

\section{Acknowledgments}

Mehedi Hasan acknowledges the Natural Sciences and Engineering Research Council of Canada (NSERC) for their support through the Vanier Canada Graduate Scholarship program. Trevor J. Hall is also grateful to the University of Ottawa for their support of a University Research Chair.

\section{Disclosures}

The authors declare no conflicts of interest.

\section{References}

1. J. Han, B. Seo, S. Ku. Kim, H. Zhang and H. R. Fetterman, "Single-chip integrated electro-optic polymer photonic RF phase shifter array," J. Lightwave Technol. 21, 3257-3261, (2003).

2. T. Niu, X. Wang, E. H. W. Chan, X. Feng and B. O. Guan, "Dual-polarization dual-parallel MZM and optical phase shifterbased microwave photonic phase controller," IEEE Photonics J. 8, 1-14, (2016).

3. S. Zhu, M. Li, X. Wang, N. Hua Zhu, and W. Li, " $1 \times \mathrm{N}$ hybrid radio frequency photonic splitter based on a dual-polarization dual-parallel Mach Zehnder modulator," Optics Comm. 431, 10-13, (2019).

4. Y. Chen, A wideband photonic microwave phase shifter with 360-degree phase tunable range based on a DP-QPSK modulator, Opt. Commun. 410, 787-792, (2018).

5. G. Li, Y. Xiang and S. Pan, "Wideband optical cancellation of RF interference with phase change," 2017 16th International Conference on Optical Communications and Networks (ICOCN), Wuzhen, 2017, pp. 1-3.

6. T. Li, E. H. W. Chan, X. Wang, X. Feng and B. Guan, "All-optical photonic microwave phase shifter requiring only a single DC voltage control," IEEE Photonics J. 8, 1-8, (2016).

7. E. H. W. Chan, W. Zhang, and R. A. Minasian, "Photonic RF phase shifter based on optical carrier and RF modulation sidebands amplitude and phase control," J. Lightwave Technol. 30, 3672-3678 (2012).

8. X. Wang, T. Niu, E. H. W. Chan, X. Feng, B. Guan and J. Yao, "Photonics-based wideband microwave phase shifter," IEEE Photonics J. 9, 1-10, (2017).

9. C. Porzi, G. Serafino, M. Sans, F. Falconi, V. Sorianello, S. Pinna, J. E. Mitchell, M. Romagnoli, A. Bogoni, and P. Ghelfi , "Photonic integrated microwave phase shifter up to the mm-wave band with fast response time in silicon-on-insulator technology," J. Lightwave Technol. 36, 4494-4500, (2018).

10. J. Dai, Z. Zhao, Y. Zeng, J. Liu, A. Liu, T. Zhang, F. Yin, Y. Zhou, Y. Liu, and K. Xu, "Stabilized optoelectronic oscillator with enlarged frequency-drift compensation range," IEEE Photon. Technol. Lett. 30, 1289-1292, (2018).

11. R. Maldonado-Basilio, M. Hasan, H, Nikkah, S. Abdul-Majid, R. Gumeri, F. Lucarz, J. Tocnaye, and T. J. Hall, "Electrooptic up-conversion mixer amenable to photonic integration," J. Mod. Optics 62, 1405-1411, (2015).

12. H. Yamazaki, T. Saida, T. Goh, A. Mori, and S. Mino, "Dual-carrier IQ modulator with a complementary frequency shifter," Opt. Express 19, B69-B74 (2011).

13. C. W. Chow, C. H. Wang, C. H. Yeh, and S. Chi, "Analysis of the carrier-suppressed single-sideband modulators used to mitigate Rayleigh backscattering in carrier-distributed PON" Opt. Express 19, 10973-10978, (2011).

14. Wen-Jr Jiang, Chun-Ting Lin, Chung-hung Ho, Chia-Chien Wei, Po-Tsung Shih, Jason (Jyehong) Chen, and Sien Chi, "Photonic vector signal generation employing a novel optical direct-detection in-phase/quadrature-phase up conversion," Opt. Lett. 35, 4069-4071 (2010).

15. H. Yamazaki, H. Takahashi, T. Goh, Y. Hashizume, T. Yamada, S. Mino, H. Kawakami, and Y. Miyamoto, "Optical modulator with a near-linear field response," J. Lightwave Technol.34, 3796-3802 (2016).

16. H. Yamazaki, T. Saida, T. Goh S. Mino, M. Nagatani, H. Nosaka, K. Murata, "Dual-carrier dual-polarization IQ modulator using a complementary frequency shifter," IEEE J. Selec. Top. Quantum Electron. 19 (6), 3400208, (2013). 